\section{Consortium nurtures ex vivo gene therapy firm}

Researchers from five research institutes have banded together to move an innovative gene therapy out of academia and into the commercial arena. Orchard Therapeutics was launched in May by a group of collaborators that reads like a who's who in gene therapyamong them Adrian Thrasher of Great Ormond, London, David Williams of Boston Children's Hospital, Don Kohn of University of California in Los Angeles and University College London's Bobby Gaspar, who will serve as the company's CSO. The company raised $\$ 33$ million series A financing from F-Prime Capital of Cambridge, Massachusetts, with support from University College London Business and Technology Fund.

Orchard's focus is on developing ex vivo autologous lentiviral stem cell gene therapies, an area in which the founding scientists and Nicolas Koebel, the company's senior vice president for business operations, are well-versed. Koebel was part of the group at GlaxoSmithKline (GSK; Brentford, UK) that forged a collaboration with San Raffaele Telethon Institute for Gene Therapy (TIGET) in Milan to develop a gene therapy for the rare severe immune deficiency ADA-SCID, characterized by a deficiency in adenosine deaminase. This therapy was the first ex vivo package presented to the European Medicines Agency, and in April, GSK and collaborators learned that it had received the go-ahead from the Committee for Medicinal Products for Human Use of the European Medicines Agency (see feature page 600).

Orchard's founding scientists have been working together since at least 2005, when they formed the Transatlantic Gene Therapy Consortium to tackle the safety problems encountered with the first generation of retroviral gene therapy vectors. This grassroots effort, which combined the expertise from a number of centers in treating rare diseases with different gene therapy platforms, was highly successful, according to Williams. In starting Orchard, the idea was to bring a biotech structure to that expertise, says Williams. "We felt the focus was correct, the timing was correct," he says. The company's role now is to get approval, negotiate reimbursement and to commercialize the therapy to make it available to patients, says Koebel. Getting reimbursement will be key for the small biotech, but Koebel is confident that when the time comes they will be able to demonstrate that the technology delivers transformative clinical benefit to patients. "Generally our stand and probably for the industry [should be] to move the discussion from a price discussion to a discussion of the value," he says. The company has an ADA-SCID program in clinical trials, with 32 patients treated so far, and has a preclinical program in mucopolysaccharidosis type III, which will be ready for the clinic later this year.

Laura DeFrancesco modification, citrullination removes a positive charge from the protein surface, a small change that can have major consequences by altering hydrogen bonding, and by modifying the protein's structure and even function. In people with certain genetic backgrounds citrullinated proteins can be immunogenic.

The study of citrullination, however, was until recently "a backwater," says Padlock's Thompson. "Nobody thought it was interesting." The rheumatology community was the exception. That's because in 1998 Dutch researchers showed that $76 \%$ of rheumatoid arthritis patients produce antibodies that recognize citrullinated autoantigens (J. Clin Invest. 101, 273-281, 1998). A commercial assay for such antibodies is now the standard for diagnosing the disease. And although no one knows what triggers the autoimmune response in rheumatoid arthritis, the autoantigens are certainly citrullinated proteins, says rheumatologist Patrick Venables of Oxford University in the UK, "and citrullinated proteins are created by PAD enzymes." Indeed, citrullinated antigens can be found in patients up to a decade before they show up in the doctor's office with clinical symptoms of rheumatoid arthritis. The hope for PAD inhibitors in rheumatoid arthritis is that removing the citrullinated antigens will resolve the disease, much like in celiac disease where eliminating the antigen (gliadin protein in dietary gluten) cures the condition.

Although this rationale has existed for at least a decade, two problems stood in the way of targeting these enzymes, says Thompson. The first was the poor understanding of the physiological role of PADs, which made side effects hard to predict, and the second was the difficulty in making specific and potent inhibitors that penetrate cells and are also metabolically stable. Of the five PADs in the enzyme family, the favorite target is PAD4, which has been genetically linked to some rheumatoid arthritis cases and whose expression is mostly limited to neutrophils. Thompson's laboratory, beginning in 2005, made a series of increasingly potent covalent pan-PAD inhibitors that he and others used over the last decade in various mouse models of autoimmune disease to demonstrate efficacy and safety. Also during this period, Brentford, UK-based GlaxoSmithKline (GSK), using a screening approach (Nat. Chem. Biol. 11, 189-192, 2015), developed a series of specific smallmolecule PAD4 inhibitors. GSK licensed these compounds to Padlock last year, and they now are in the hands of BMS. These compounds, by showing specificity with noncovalent binding, which most pharma consider to be theoretically safer, "reinvigorated interest in the field," says Thompson.

Padlock's drugs block citrullination taking place inside and outside cells. In the course of rheumatoid arthritis, neutrophils in inflamed joints release PAD4 into synovial fluid, where calcium concentrations are high. Because PAD enzymes are calciumdependent, this adds fuel to the fire by sending PADs hunting for proteins to citrullinate, creating more autoantigens in a feed-forward inflammatory loop. But PADs also citrullinate histones, transcription factors and other proteins inside cells, which may help drive disease.

Whether PAD inhibitors will still be effective once rheumatoid arthritis is established remains unknown, because advanced disease is multifaceted. In animals PAD inhibitors are effective both in preventing disease and after disease onset, says Thompson. These results suggest PAD activity continues late in the disease process and is worth targeting. Also, Venables recently used a pan-PAD inhibitor to treat mice with collagen-induced arthritis, a standard rheumatoid arthritis model, and at a high dose, the drug on its own completely halted the progression of the disease (the work has not yet been published).

But Venables hesitates to predict success for PAD inhibitors in rheumatoid arthritis. PADs are promiscuous and citrullinate many other proteins. "The worry is going to be side effects, isn't it?" he says. "Because there are important physiological functions [of PADs]." In two 2004 papers (Cell 118, 545-553, 2004; Science 306, 279-283, 2004) researchers reported that PAD4 citrullinates histones and this could turn off gene expression by antagonizing histone methylation. So inhibiting PAD4 might have unpredictable effects on the expression of multiple genes. Thompson says tumor formation is unlikely, because in cells and animals PADs generally promote oncogenesis, not the reverse.

On the plus side, inside cells, PAD activity appears to be well controlled, and levels are generally very low, Thompson says. And 